\title{
FORENSIC VALUE OF TEN SHORT TANDEM REPEAT LOCI IN TURKEY COMPARED TO OTHER ETHNIC GROUPS
}

\author{
Ozkorkmaz A ${ }^{1}$, Baransel Isir $\mathrm{A}^{2, *}$, Pehlivan $\mathrm{S}^{3,4}$. Gökalp Özkorkmaz E
}

*Corresponding Author: Aysun Baransel Isir, Department of Forensic Medicine, School of Medicine, University of Gaziantep, 27100 Gaziantep, Turkey; Tel.: +90-532-701-4712; Fax: +90342-338-5000; E-mail: aybaranse1@yahoo.com

\begin{abstract}
Allele frequencies of the 10 short tandem repeats (STRs) loci (D16S539, D2S1338, D3S1358, vWA, D18S51, D21S11, D8S1179, D19S433, FGA, THO1) included in the AmpFISTR SGM Plus kit, were obtained from biological samples from 100 unrelated individual residing in different part of Turkey. The $\chi 2$ test showed that all these loci agreed with Hardy-Weinberg equilibrium, The results were compared with the previously published data from Turkish and other ethnic groups. Suggest that these loci with their high heterozygosity and combined power of discrimination (PD) values are useful for forensic identifications.
\end{abstract}

Keywords: Forensic science; AmpFISTR SGM Plus; Short tandem repeats (STRs); Population genetics; Turkey

1 Department of Biology, Ege University, Faculty of Science, Bornova-Izmir, Turkey

2 Department of Forensic Medicine, Gaziantep University, Faculty of Medicine, Gaziantep, Turkey

3 Genetic Disease and Application Center, Ege University, Faculty of Science, Bornova-Izmir, Turkey

4 Department of Medical Biology and Genetics, Gaziantep University, Faculty of Medicine, Gaziantep, Turkey

5 College of Health, Ahi Evran University, Kýrsehir, Turkey
Subjects. Samples were obtained from 100 unrelated Turkish individuals living in Turkey (12 females, 88 males). Individuals were selected from seven geographical region of Turkey. From the Marmara Region (11 individuals), Aegean Region (17 individuals), Central Anatolian Region (24 individuals), Eastern Anatolian Region (16 individuals), Black Sea Region (16 individuals), Mediterranean Region (seven individuals) and Southeastern Region (nine individuals) (Figure 1).

DNA Extraction and Typing. Genomic DNA was isolated from blood, tissue, saliva, hair and semen samples using the phenol-chloroform isoamyl alcohol method [1] [AmpFlSTR SGM Plus ${ }^{\mathrm{TM}}$ Polymerase Chain Reaction (PCR) Amplification Kit user manual, 2001]. Multiplex PCR, using 1-2 ng target DNA, was performed with the GeneAmp PCR system 9700 using the AmpFlSTR SGM Plus $^{\text {TM }}$ kit (Applied Biosystems Division/PerkinElmer, Foster City, CA, USA) according to the manufacturer's instructions. The loci D16S539, D2S1338, D3S1358, vWA, D18S51, D21S11, D8S1179, D19S433, FGA, THO1 were co-amplified and analyzed on ABI PRISM 3100 Genetic analyzer. The results were analyzed using Gene Scan analysis version 3.1.2 software and genotypes were determined by comparison to allelic ladder using Genotyper DNA fragment analysis version 2.0 software (Applied Biosystems/Perkin Elmer).

Analysis of Data. Evaluation of the HardyWeinberg equilibrium expectations was carried out using the Fisher's exact $t$-test. Further calculation 


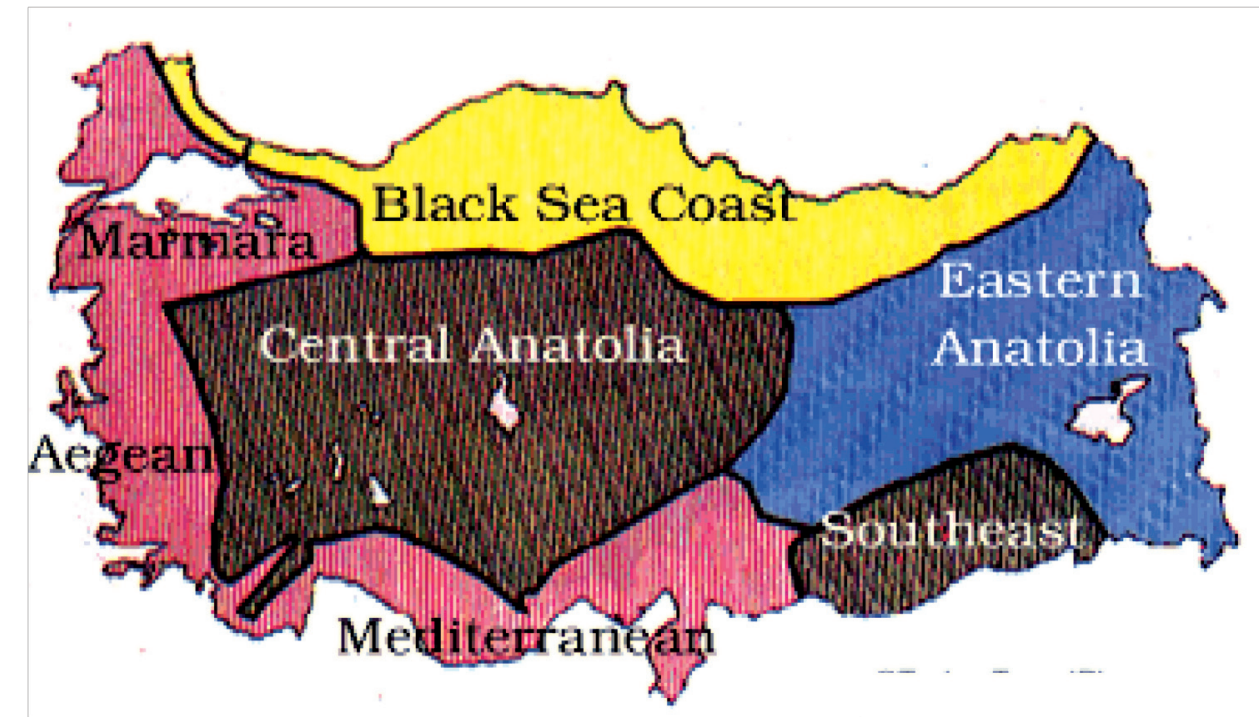

Fig. 1. Map of Turkey showing its all geographical regions.

of statistical parameters of forensic interest and population differentiation tests were conducted using the Arlequin 2.0 software package [2].

Results and discussion: Allele frequencies for 10 short tandem repeat (STR) loci and parameters of genetic and forensic interest are presented in Table 1. In the Turkish population, the D3S1358 locus allele 16 (0.556), vWA locus allele 17 (0.486), D16S539 locus allele 11 (0.555), D2S1338 locus allele 17 (0.412), D8S1179 locus allele 13 (0.483), D21S11 locus allele 30 (0.407), D18S51 locus allele 14 (0.349), D19S433 locus allele 14 (0.385), THO1 locus allele 6 (0.604), FGA locus allele $22(0.460)$, are the most common alleles. The frequencies ranged from 0.005 to 0.604 . The Fisher's exact $t$-test results indicated that the loci were in Hardy-Weinberg equilibrium. The most discriminating loci, according to their power of exclusion (PE) values, were D2S1338 and D18S51, whereas THO1 and D3S1358 turned out to be the least discriminating loci. The heterozygosity of the 10 STR loci screened in this study ranged from 0.77 to 0.89 , indicating that these loci could be used in determination of identity because of the high heterozygosity. The 10 loci have a pow- er of discrimination (PD) 0.99999999999988 and the combined power of exclusion (PE) is 0.999994 in Turkey. The probability of identity across all 10 loci was estimated as 1 in 8.33 trillion. Thus, the PD value estimated for the Turkish population was highly significant.

On comparison of the observed allele frequencies with previously published data from Turkey [3-9], we found no statistically significant difference for all STRs $(p>0.05)$. Allele 22 of vWA and allele 9 of the D19S433 locus were detected in Turkish individuals, and are absent in Chinese, North and West African and Czech populations [10-13]. The observed combined PD and PE values were also compared with the published populations [10-13]. The combined PE value (0.999994) for Turks was higher than for Chinese (0.999949), Czech (0.999971) and North African (0.999522) populations, but lower than for West Africans (0.99999946). The combined PD value in the Turkish population also seems to be a little higher than in the Chinese (0.999999999997) and the Czech (0.99999999999939), and a little lower than in North African (0.99999999999999959) and West African (0.99999999999999999952) populations. 
BALKAN JOURNAL OF MEDICAL GENETICS

${\text { Ozkorkmaz A }{ }^{1} \text {, Baransel Isir A }{ }^{2, *} \text {, Pehlivan } \mathrm{S}^{3,4} \text {. Gökalp Özkorkmaz E }}^{5}$

Table 1. Observed allele frequencies for AmpFISTR SGM Plus loci in a sample of 100 individuals from Turkey

\begin{tabular}{|c|c|c|c|c|c|c|c|c|c|c|}
\hline Allele & D16S539 & D2S1338 & D3S1358 & vWA & D18S51 & D21S11 & D8S1179 & D19S433 & FGA & THO1 \\
\hline 6 & & & & & & & & & & 0.604 \\
\hline 7 & & & & & & & & & & 0.098 \\
\hline 8 & 0.015 & & & & & & 0.009 & & & 0.020 \\
\hline 9 & 0.056 & & & & & & 0.005 & 0.011 & & 0.222 \\
\hline 9.3 & & & & & & & & & & 0.046 \\
\hline 10 & 0.028 & & & & 0.006 & & 0.044 & & & 0.009 \\
\hline 10.2 & & & & & 0.008 & & & & & \\
\hline 11 & 0.555 & & & & 0.020 & & 0.015 & 0.008 & & \\
\hline 12 & 0.228 & & & & 0.191 & & 0.071 & 0.085 & & \\
\hline 13 & 0.110 & & 0.007 & 0.009 & 0.124 & & 0.483 & 0.200 & & \\
\hline 13.2 & & & & & 0.005 & & & 0.015 & & \\
\hline 14 & 0.007 & & 0.030 & 0.026 & 0.349 & & 0.220 & 0.385 & & \\
\hline 14.2 & & & & & & & & 0.042 & & \\
\hline 15 & & & 0.228 & 0.072 & 0.064 & & 0.124 & 0.126 & & \\
\hline 15.2 & & & & & & & & 0.059 & & \\
\hline 16 & & 0.009 & 0.556 & 0.122 & 0.088 & & 0.025 & 0.031 & & \\
\hline 16.2 & & & & & & & & 0.022 & & \\
\hline 17 & & 0.412 & 0.228 & 0.486 & 0.048 & & & 0.005 & & \\
\hline 17.2 & & & & & & & & 0.006 & & \\
\hline 18 & & 0.055 & 0.058 & 0.218 & 0.036 & & & & 0.006 & \\
\hline 19 & & 0.125 & 0.014 & 0.043 & 0.027 & & & & 0.019 & \\
\hline 20 & & 0.081 & & 0.016 & 0.011 & & & & 0.030 & \\
\hline 21 & & 0.019 & & & 0.015 & & & & 0.124 & \\
\hline 22 & & 0.038 & & 0.006 & 0.005 & & & & 0.460 & \\
\hline 23 & & 0.205 & & & & & & & 0.218 & \\
\hline 24 & & 0.027 & & & & & & & 0.075 & \\
\hline 25 & & 0.013 & & & & & & & 0.046 & \\
\hline 26 & & 0.006 & & & & & & & 0.012 & \\
\hline 27 & & & & & & 0.013 & & & 0.006 & \\
\hline 28 & & 0.005 & & & & 0.128 & & & & \\
\hline 29 & & & & & & 0.211 & & & & \\
\hline 30 & & & & & & 0.406 & & & & \\
\hline 30.2 & & & & & & 0.025 & & & & \\
\hline 31 & & & & & & 0.018 & & & & \\
\hline 31.2 & & & & & & 0.083 & & & & \\
\hline 32 & & & & & & 0.005 & & & & \\
\hline 32.2 & & & & & & 0.038 & & & & \\
\hline 33 & & & & & & 0.006 & & & & \\
\hline 33.2 & & & & & & 0.056 & & & & \\
\hline 34.2 & & & & & & 0.009 & & & & \\
\hline $\mathbf{H}$ & 0.82 & 0.85 & 0.77 & 0.85 & 0.89 & 0.78 & 0.80 & 0.82 & 0.80 & 0.80 \\
\hline PD & 0.9314 & 0.9647 & 0.9066 & 0.9336 & 0.9610 & 0.9612 & 0.9444 & 0.9392 & 0.9552 & 0.9656 \\
\hline $\mathbf{P E}$ & 0.6698 & 0.7687 & 0.5847 & 0.6669 & 0.7578 & 0.7361 & 0.6952 & 0.6970 & 0.7199 & 0.6387 \\
\hline MP & 0.0686 & 0.0353 & 0.0934 & 0.0664 & 0.0390 & 0.0388 & 0.0556 & 0.0608 & 0.0448 & 0.0844 \\
\hline PIC & 0.9990 & 0.9998 & 0.9979 & 0.9991 & 0.9998 & 0.9997 & 0.9993 & 0.9994 & 0.9996 & 0.9985 \\
\hline $\mathbf{P}$ & 0.8244 & 0.3033 & 0.9512 & 0.9064 & 0.5108 & 0.6878 & 0.8306 & 0.3858 & 0.8790 & 0.7152 \\
\hline
\end{tabular}

H: Heterozygosity; PD: power of discrimination; PE: power of exclusion; MP: matching probability; PIC: polymorphism information content; P: Hardy-Weinberg equilibrium exact test based on 3100 shufflings 
In conclusion, our results show that 10 STR loci (D16S539, D2S1338, D3S1358, vWA, D18S51, D21S11, D8S1179, D19S433, FGA, THO1) are useful markers for personal identification and forensic case work in the Turkish population.

\section{REFERENCES}

1. Sambrook J, Fritsch EF, Maniatis T. Molecular Cloning: a Laboratory Manual. Cold Spring Harbor: Cold Spring Harbor Laboratory Press, 1989.

2. Schneider S, Roessli SD, Exoffier L, A software for population genetics data analysis, Arlequin version 2.0, Genetics and Biometry Laboratory, University of Geneva, Geneva, Switzerland, 2000.

3. Asicioglu F, Akyuz F, Cetinkaya U, Ozbek U. Allele distribution data of nine short tandem repeat loci for Turkish population: D3S1358, vWa, FGA, D8S1179, D21S11, D18S51, D13S317, D7S820. Forensic Sci Int 2002; 129(1): 75-77.

4. Cakir AH, Celebioglu A, Altunbas S. STR data for the AmpFISTR SGM Plus from Marmara region of Turkey. Forensic Sci Int 2002; 127(3): 240-242.

5. Cakir AH, Celebioglu A, Simsek F. STR data for the AmpFISTR SGM Plus from Agean region of Turkey. Forensic Sci Int 2002; 129(2): 137-139.

6. Cakir AH, Celebioglu A, Altunbas S, Yardimci E. Allele frequencies for 15 STR loci in Van-Agri districts of the Eastern Anatolia region of Turkey. Forensic Sci Int 2003; 135(1): 60-63.
7. Cakir AH, Simsek F. Katirci N, Tasdelen B. STR data for the AmpFISTR SGM Plus from the eastern and western sections of Mediterranean region of Turkey. Forensic Sci Int 2004; 142(1): 5557.

8. Filoglu G, Kalfaoglu AE, Atasoy S. Allele frequencies for 10 STR loci in Istanbul (Turkey) population. J Forensic Sci 2002; 47(4): 909-910.

9. Erkol Z. Tug A, Alakoc DY, Elma C, Buken B, Cetinyurek A, Erkol H. STR data for the AmpFISTR identifier loci from an old settlement in northwestern Turkey. Forensic Sci Int 2007; 173(23): 238-240.

10. Cai Y, Xiang D, Liang X, Xu Y, Zhuang Y. STR data for the AmpFISTR SGM Plus loci from Shantou (South of China). Forensic Sci Int 2003; 132(3): 225-227.

11. Gonçalves R, Jesus J, Fernandes AT, Brehm A. Genetic profile of a multi-ethnic population from Guine-Bissau (West African coast) using the new PowerPlex 16 System kit. Forensic Sci Int 2002; 129(1): 78-80.

12. Picornell A, Tomas C, Jimenez G, Castro JA, Ramon MM. Jewish population genetic data in 20 polymorphic loci. Forensic Sci Int 2002; 125(1): 52-58.

13. Vanek D, Hradil R, Budowle B. Czech population data on 10 short tandem repeat loci of SGM Plus STR system kit using DNA purified in FTA cards. Forensic Sci Int 2001; 119(1): 107-108. 